\title{
Sur la convergence d'une série d'opérateurs
}

par

B. STANKOVIC (Novi Sad)

Dans le corps des opérateurs introduits par J. Mikusiński [1] nous étudions les conditions de convergence pour la série:

$$
\sum_{i=0}^{\infty} \gamma_{i} \lambda^{i} s^{i a}, \quad \alpha>0,
$$

où $\lambda \neq 0$ et $\gamma_{i}$ sont des nombres complexes.

C. Ryll-Nardzewski [2] a traité cette série pour $\alpha=1$, et pour $0<\alpha<1, \gamma_{i}=1 / i$ !. Avec la même méthode on a, pour la série (1) le théorème suivant qui contient, pour $\alpha=1$, celui de Ryll-Nardzewski: THÉORÈME. S'il existe un nombre $\delta>a$ tel que

$$
\lim \sup n^{\delta n}\left|\gamma_{n}\right|<\infty,
$$

la série (1) converge pour tout $\lambda$ complexe.

si, au contraire, il existe un nombre $\eta>0$ tel que

$$
\left|\gamma_{n}\right| \Gamma(n \alpha) \geqslant \eta^{n}, \quad n \geqslant n_{0}
$$

la série considérée diverge pour tout $\lambda \neq 0$.

Démontrons d'abord le lemme suivant:

LEMME. Supposons que pour tout $i_{0} \in N$ il existe au moins un $i \geqslant i_{0}$ tel que $\gamma_{i} \neq 0$. Alors, pour que

$$
\sigma_{n}=\sum_{i=0}^{n} \gamma_{i} \lambda^{i} s^{i a} f, \quad f \in O,
$$

soit une suite appartenant d' l'anneau des fonctions. continues $O$ [1] il faut et il suffit que $f^{(i)}$ existe et que $f^{(i)}(0)=0$ pour tout $i \in N$.

Démonstration. Des relations

$$
\sigma_{1}=\gamma_{0} f+\gamma_{1} \lambda s^{a} f, \quad \sigma_{n}=\sigma_{n-1}+\gamma_{n} \lambda^{n} s^{n a} f, \quad n \geqslant 2,
$$


on déduit que, s'il existe une fonction $f \in C$ telle que $\left(\sigma_{n}\right) \subset C$, on a. $8^{\text {na }} f$ $=g_{n} \epsilon C$ ou $\gamma_{n}=0$. D'après l'hypothèse, il existe, pour tout $i_{0} \in N$, au moins un $i \geqslant i_{0}$ tel que $s^{i a} f=g_{i} \epsilon O$, c'est-à-dire

$$
f=l^{i a} g_{i}=\left\{\int_{0}^{t} \frac{(t-u)^{i \alpha-1}}{\Gamma(i \alpha)} g_{i}(u) d u\right\}
$$

d'où les propriétés de $f$. D'autre part, si $f$ a les propriétés mentionées, alors $s^{k a} f=l^{k(p-a)+1} f^{(p k+1)} \epsilon O, \quad p \in N, \quad p>\alpha$, et le lemme est démontré.

Démonstration du théorème. Considérons la fonction

$$
f(\mu, t)=\int_{-i \infty}^{i \infty} z^{\mu} \exp \left(z t-z^{\sigma}\right) d z, \quad 0<\sigma<1 .
$$

On a $s^{n a}\{f(0, t)\}=\{f(n \alpha, t)\}, f^{(i)}(0,0)=0, i \in N$, et

$$
|f(n a, t)| \leqslant \frac{2}{\sigma}\left(\cos \frac{\sigma \pi}{2}\right)^{-(n a+1) / \sigma} \Gamma\left(\frac{n a+1}{\sigma}\right) .
$$

Reprenons la série (1)

$$
\sum_{n=0}^{\infty} \gamma_{n} \lambda^{n} s^{n \alpha}\{f(0, t)\}=\sum_{n=0}^{\infty} \gamma_{n} \lambda^{n}\{f(n \alpha, t)\}
$$

En utilisant la majoration pour $f(n a, t)$ et l'hypothèse du théorème pour $\sigma$ choisi tel que $\delta>\alpha / \sigma$, la série numérique

$$
\sum_{n=0}^{\infty} \gamma_{n} \lambda^{n} f(n \alpha, t)
$$

converge uniformément dans tout intervalle $\left[0, t_{0}\right]$.

Pour la deuxième partie du théorème supposons que $f(t)$ ne soit identiquement 0 dans aucun entourage de zéro et que la série $\sum_{n=0}^{\infty} \gamma_{n} \lambda^{n} s^{n a} f$ converge uniformément dans tout intervalle fermé $\left[0, t_{0}\right]$ pour un $\lambda$ fixe. Alors $\gamma_{n} \lambda^{n} s^{n \alpha} \underset{n \rightarrow \infty}{\rightarrow} 0$ uniformément dans tout intervalle $\left[0, t_{0}\right]$.

D'après le lemme on a $f^{(i)}(0)=0, i \in N$, et la formule de Taylor pour $f(t)$ donne:

$$
f(t)=\frac{t^{n}}{n !} f^{(n)}(\theta t), \quad 0<\theta<1
$$

Soit $k_{n} \in N$ et tel que $0<k_{n} \alpha-n=r_{n} \leqslant \alpha$, et $p>\alpha, p \in N$, alors

$$
f(t)=\frac{t^{n}}{n !} \int_{0}^{\theta t} \frac{(\theta t-u)^{k_{n} a-n-1}}{\Gamma\left(k_{n} \alpha-n\right)} d u \int_{0}^{u} \frac{(u-\tau)^{k_{n}(p-\alpha)}}{\Gamma\left(k_{n} p-k_{n} \alpha+1\right)} f^{\left(k_{n} p+1\right)}(\tau) d \tau,
$$

où

$$
\left\{\int_{0}^{t} \frac{(t-\tau)^{k_{n}(p-a)}}{\Gamma\left(k_{n} p-k_{n} a+1\right)} f^{\left(k_{n} p+1\right)}(\tau) d \tau\right\}=s^{k_{n} a} f .
$$

On sait que $\left|\gamma_{n} \lambda^{n} s^{n \alpha} f\right|<\varepsilon, n \geqslant n_{0}, 0 \leqslant t \leqslant t_{0}$. En vertu de l'hypothèse du théorème, on a la majoration:

$$
\begin{aligned}
|f(t)| & \leqslant \frac{\varepsilon t_{0}^{k_{n}{ }^{\alpha}}}{|\lambda|^{k_{n}}\left|\gamma_{k_{n}}\right|} \frac{1}{\Gamma\left(k_{n} \alpha-r_{n}+1\right) \Gamma\left(r_{n}+1\right)}, \quad 0 \leqslant t \leqslant t_{0}, \\
& \leqslant K\left(\frac{t_{0}^{\alpha}}{|\lambda| \eta}\right)^{k_{n}}\left(k_{n}\right)^{r_{n}-1}, \quad K \text { étant une constante. }
\end{aligned}
$$

Quand $t_{0}^{a}<|\lambda| \eta$, la fonction $f(t)$ est identiquement zéro pour $0 \leqslant t$ $\leqslant t_{0}$. Cela est en contradiction avec l'hypothèse faite sur $f(t)$, et la série (1) ne converge poür aucune valeur de $\lambda$.

Voici encore une propriété intéressante de la série (1):

Propositron. Si la série (1) converge dans le corps des opérateurs pour $\lambda=\lambda_{0} \neq 0$, elle converge aussi pour tout $\lambda$.

Démonstration. Si la série converge pour $\lambda=\lambda_{0}$, il existe une fonction $f_{\epsilon} C$ telle que $\sum_{k=0}^{\infty} \gamma_{k} \lambda^{k} s^{k a} f$ est une série d'éléments de $C$ qui converge unformément dans tout intervalle fermé $0 \leqslant t \leqslant t_{0}$. D'après le lemme on a pour $p \in N^{*}$ et $p>a$ :

$$
\sum_{k=0}^{\infty} \gamma_{k} \lambda_{0}^{k} s^{k a} f=\sum_{k=0}^{\infty} \gamma_{k} \lambda_{0}^{k}\left\{\int_{0}^{t} \frac{(t-u)^{k(p-\alpha)}}{\Gamma(k p-k \alpha+1)} f^{(k p+1)}(u) d u\right\} .
$$

Soit $\omega>0, w=(1 / \omega)^{1 / a}$ et $F^{\prime}(u)=f(w u)$. Nous allons montrer que la série $\sum_{k=0}^{\infty} \gamma_{k}\left(\lambda_{0} \omega\right)^{k} s^{k a}$ converge dans le corps des opérateurs:

$$
\sum_{k=0}^{\infty} \gamma_{k}\left(\lambda_{0} \omega\right)^{k} s^{k \alpha} F=\sum_{k=0}^{\infty} \gamma_{k}\left(\lambda_{0} \omega\right)^{k} \int_{0}^{t} \frac{(t-u)^{k(p-\alpha)}}{\Gamma(k p-k \alpha+1)} F^{(k p+1)}(u) d u .
$$

Pour $x=w t$ on a

$$
=\sum_{k=0}^{\infty} \gamma_{k} \lambda_{0}^{k} \int_{0}^{x} \frac{(x-u)^{k(p-\alpha)}}{\Gamma(k p-k \alpha+1)} f^{(k p+1)}(u) d u
$$


et cette série converge uniformément dans tout intervalle fermé $\left[0, x_{0}\right]$. La série précédente converge uniformément dans tout intervalle $\left[0, x_{0} / \omega\right]$. Mais elle converge aussi pour tout $|\lambda|<\omega\left|\lambda_{0}\right|$.

\section{Travaux cités}

[1] J. Mikusiński, Operational calculus, New York - London - Paris 1959. [2] C. Ryll-Nardzewski, Sur la convergence des séries de puissances de l'opérateur différentiel, Studia Math. 13 (1953), p. 37-40.

Regu par la Rédaction le 3. 10. 1964

\section{On integrals of functions \\ with values in a complete linear metric space}

by

D. PRZEWORSKA-ROLEWICZ and S. ROLEWICZ (WarsZaWa)

In paper [5] S. Mazur and W. Orlicz gave a definition of a Riemann integral of a function $x(t)$ determined on a set in a Euclidean space with values in a complete linear metric space $X$ ( $F$-space in Banach's terminology [3]). In that paper it was shown that the space $X$ is locally convex if and only if each continuous function $x(t)$ is integrable.

In this note some conditions of the existence of a Riemann integral will be given. This will permit us to extend the integral method of Banach algebras [4], [5] to the theory of locally bounded algebras [11], [12]. As a particular case, the existence of analytic functions of many elements will be proved.

At the end of the paper we will show what difficulties arise concerning the definition of the Lebesgue-Bochner integral in the case where $X$ is not a locally convex space.

1. Let $L$ be a set in the $n$-dimensional Euclidean space $E^{n}$. And let $|\cdot|$ be some measure determined on $L$. We will assume that the measure of the whole $L$ is finite.

By a partition $\Delta$ we shall mean a decomposition of the set $L$ into a union of closed sets $L=\bigcup_{i=1}^{n} L_{i}$ such that $\left|L_{i} \cap L_{j}\right|=0$ for $i \neq j$. We will write $\Delta=\left(L_{1}, \ldots, L_{n}\right)$. We say that the sequence of partitions $\Delta^{j}=\left(L_{1}^{j}, \ldots, L_{n}^{j}\right)$ is normal if the largest diameter of $L_{i}^{j}$ tends to 0 :

$$
\lim _{j \rightarrow \infty} \sup _{1 \leqslant i \leqslant n_{j}} \sup _{t, t_{1} \in L_{i}^{j}}\left|t-t_{1}\right|=0 .
$$

Let $X$ be a complete linear metric space. Let $x(t)$ be a function determined on $L$ with values in $X$. Let $\Delta^{j}=\left(L_{1}^{j}, \ldots, L_{n_{j}}^{j}\right)$ be a normal sequence of partitions. Let $t_{i}^{j} \epsilon L_{i}^{j}$. We write

$$
S\left(x, \Delta^{j}, t_{i}^{j}\right)=\sum_{i=1}^{n_{j}} x\left(t_{i}^{j}\right)\left|L_{i}^{j}\right| .
$$

